\title{
On the Weyl Representation of Metaplectic Operators
}

\author{
Maurice A de Gosson \\ Universität Potsdam, Institut f. Mathematik \\ Am Neuen Palais 10, D-14415 Potsdam \\ maurice.degosson@gmail.com
}

August 13, 2018

\begin{abstract}
We study the Weyl representation of metaplectic operators associated to a symplectic matrix having no non-trivial fixed point, and justify a formula suggested in earlier work of Mehlig and Wilkinson. We give precise calculations of the associated Maslov-type indices; these indices intervene in a crucial way in Gutzwiller's formula of semiclassical mechanics, and are simply related to an index defined by Conley and Zehnder.
\end{abstract}

Received 26 December 2004, revised 18 February 2005

MSC 2000: 81S30, 43A65, 43A32

Keywords: Weyl symbol, metaplectic operators, Maslov and Conley-Zehnder index, Gutzwiller formula

\section{Introduction}

In a remarkable paper [14] Mehlig and Wilkinson propose a simple derivation of Gutzwiller's 9] approximation

$$
\widetilde{\rho}_{\text {Gutz }}(E)=\frac{1}{\pi \hbar} \operatorname{Re} \sum_{p o} \frac{T_{p o} i^{\nu_{p o}}}{\sqrt{\left|\operatorname{det}\left(S_{p o}-I\right)\right|}} e^{i \mathcal{A}_{p o} / \hbar}
$$

for the oscillating part of the semiclassical level density for chaotic systems whose periodic orbits " $p o$ " are all isolated and non-degenerate $\left(T_{p o}\right.$ is the prime period, $\nu_{p o}$ an integer related to the Maslov index, $\mathcal{A}_{p o}$ the action, and $S_{p o}$ the stability matrix). Mehlig and Wilkinson's derivation heavily relies upon their observation that for any symplectic matrix $S$ such that $\operatorname{det}(S-I) \neq 0$ one has

$$
\frac{i^{\nu}}{\sqrt{|\operatorname{det}(S-I)|}}=\operatorname{Tr}\left[\widehat{R}_{\nu}(S)\right]
$$


where $\widehat{R}_{\nu}(S)$ is the operator $L^{2}\left(\mathbb{R}_{x}^{n}\right) \longrightarrow L^{2}\left(\mathbb{R}_{x}^{n}\right)$ defined by

$$
\widehat{R}_{\nu}(S) \Psi(x)=\left(\frac{1}{2 \pi}\right)^{n} \frac{i^{\nu(S)}}{\sqrt{|\operatorname{det}(S-I)|}} \int e^{\frac{i}{2}\left\langle M_{S} z_{0}, z_{0}\right\rangle} \widehat{T}\left(z_{0}\right) \Psi(x) d^{2 n} z_{0}
$$

where:

- $\widehat{T}\left(z_{0}\right)$ is the Weyl-Heisenberg operator associated to $z_{0}=\left(x_{0}, p_{0}\right) \in \mathbb{R}^{2 n}$ :

$$
\widehat{T}\left(z_{0}\right) f(x)=e^{i\left(\left\langle p_{0}, x\right\rangle-\frac{1}{2}\left\langle p_{0}, x_{0}\right\rangle\right)} f\left(x-x_{0}\right)
$$

for any function $f$ defined on $\mathbb{R}^{2 n}$;

- $M_{S}$ is a symmetric matrix, associated to $S$ by the formula

$$
M_{S}=\frac{1}{2} J(S+I)(S-I)^{-1}
$$

$I$ being the $2 n \times 2 n$ identity matrix and $J$ the standard symplectic matrix;

- The integer $\nu(S)$ corresponds to a choice of $\arg \operatorname{det}(S-I)$.

In what follows we will write Mehlig-Wilkinson's formula as a Bochner integral

$$
\widehat{R}_{\nu}(S)=\left(\frac{1}{2 \pi}\right)^{n} \frac{i^{\nu(S)}}{\sqrt{|\operatorname{det}(S-I)|}} \int e^{\frac{i}{2}\left\langle M_{S} z_{0}, z_{0}\right\rangle} \widehat{T}\left(z_{0}\right) d^{2 n} z_{0} .
$$

The validity of Mehlig and Wilkinson's derivation requires that -as these authors claim- $\widehat{R}_{\nu}(S)$ is one of the two metaplectic operators $\pm \widehat{S}$ associated with the symplectic matrix $S$. To sustain this claim the authors refer to previous work of one of the authors [16]; they also claim that for all $S, S^{\prime}$ such that $\operatorname{det}(S-I) \neq 0, \operatorname{det}\left(S^{\prime}-I\right) \neq 0$ and $\operatorname{det}\left(S S^{\prime}-I\right) \neq 0$ their operators satisfy

$$
\widehat{R}_{\nu^{\prime \prime}}\left(S S^{\prime}\right)= \pm \widehat{R}_{\nu}(S) \widehat{R}_{\nu^{\prime}}(S) .
$$

The purpose of this paper is to fully justify Mehlig and Wilkinson's statements. There are actually several options available. The a priori most direct strategy would be to use Howe's theory [10] of operators with Gaussian kernels (the "oscillator semigroup" theory: see [3] for a review); another approach would be to use Hannabuss' character theory [8] for contact transformations (also see the follow-up [1] to this paper for interesting applications to starproducts). These two methods however both have, drawbacks. Howe's theory needs quite a lot of technical prerequisites and would lead here to unnecessarily long calculations; in addition it would not be very helpful for the study 
of the sign ambiguity in (4) since this point is not really addressed in Howe's work. On the other hand, Hannabuss' machinery is quite abstract (it makes a heavy use of group character theory) and the use of this approach would perhaps have a tendency to obscure things. For these reasons we prefer a more straightforward line of attack, using standard Weyl calculus together with the theory of the metaplectic group as developed in [4. This approach moreover has, as we will see, the overwhelming advantage of producing simple formulae relating the integer $\nu$ in (3) to the usual Maslov index of the metaplectic group (we emphasize that $\nu$ is not the Maslov index!). This is important, because Gutzwiller's theory has been plagued since its very beginning by the question of how to calculate the indices $\nu_{p o}$ appearing in the trace formula (11), as witnessed by the abundant literature devoted to this delicate topic (see [7] where we discuss these issues and give a rather exhaustive list of references).

\section{Notations}

We denote by $\sigma$ the canonical symplectic form on the phase space $\mathbb{R}_{z}^{2 n}=$ $\mathbb{R}_{x}^{n} \times \mathbb{R}_{p}^{n}$ :

$$
\sigma\left(z, z^{\prime}\right)=\left\langle p, x^{\prime}\right\rangle-\left\langle p^{\prime}, x\right\rangle \text { if } z=(x, p), z^{\prime}=\left(x^{\prime} p^{\prime}\right)
$$

that is, in matrix form

$$
\sigma\left(z, z^{\prime}\right)=\left\langle J z, z^{\prime}\right\rangle \quad, \quad J=\left[\begin{array}{cc}
0 & I \\
-I & 0
\end{array}\right] .
$$

The real symplectic group $S p(n)$ consists of all linear automorphisms $S$ of $\mathbb{R}_{z}^{2 n}$ such that $\sigma\left(S z, S z^{\prime}\right)=\sigma\left(z, z^{\prime}\right)$ for all $z, z^{\prime}$. It is a connected Lie group and $\pi_{1}(\operatorname{Sp}(n))$ is isomorphic to $(\mathbb{Z},+)$. $\mathcal{S}\left(\mathbb{R}^{n}\right)$ is the Schwartz space of rapidly decreasing functions on $\mathbb{R}^{n}$ and its dual $\mathcal{S}^{\prime}\left(\mathbb{R}^{n}\right)$ the space of tempered distributions.

We will denote by Inert $R$ the number of negative eigenvalues of a real symmetric matrix $R$.

\section{Prerequisites}

In this Section we briefly recall the main definitions and properties of the metaplectic group and of Weyl calculus we will need in the rest of this paper.

\subsection{Standard theory of $M p(n)$ : Review}

The material of this first subsection is quite classical; see for instance [3, 4, 5] and the references therein. 
Every $\widehat{S} \in M p(n)$ is the product of two "quadratic Fourier transforms", which are operators $\widehat{S}_{W, m}$ defined on $\mathcal{S}\left(\mathbb{R}^{n}\right)$ by

$$
\widehat{S}_{W, m} f(x)=\left(\frac{1}{2 \pi i}\right)^{n / 2} i^{m} \sqrt{|\operatorname{det} L|} \int e^{i W\left(x, x^{\prime}\right)} f\left(x^{\prime}\right) d^{n} x^{\prime}
$$

where $W$ is a quadratic form in the variables $x, x^{\prime}$ of the type

$$
W\left(x, x^{\prime}\right)=\frac{1}{2}\langle P x, x\rangle-\left\langle L x, x^{\prime}\right\rangle+\frac{1}{2}\left\langle Q x^{\prime}, x^{\prime}\right\rangle
$$

with $P=P^{T}, Q=Q^{T}$, $\operatorname{det} L \neq 0$. The integer $m$ ("Maslov index") appearing in (5) corresponds to a choice of $\arg \operatorname{det} L$ :

$$
m \pi \equiv \arg \operatorname{det} L \bmod 2 \pi
$$

and to every $W$ there thus corresponds two different choices of $m$ modulo 4 : if $m$ is one choice, then $m+2$ is the other, reflecting the fact that $M p(n)$ is a two-fold covering of $S p(n)$. The projection $\pi: M p(n) \longrightarrow S p(n)$ is entirely specified by the datum of each $\pi\left(\widehat{S}_{W, m}\right)$, and we have $\pi\left(\widehat{S}_{W, m}\right)=S_{W}$ where

$$
(x, p)=S_{W}\left(x^{\prime}, p^{\prime}\right) \Longleftrightarrow p=\partial_{x} W\left(x, x^{\prime}\right) \text { and } p^{\prime}=-\partial_{x^{\prime}} W\left(x, x^{\prime}\right) .
$$

Rewriting these conditions in terms of $P, L, Q$ we get $p=P x-L^{T} x^{\prime}$ and $p^{\prime}=L x-Q x^{\prime}$; solving these equations in $x$ and $p$ yields

$$
x=L^{-1}\left(p^{\prime}+Q x^{\prime}\right) \text { and } p=\left(P L^{-1} Q-L^{T}\right) x^{\prime}+P L^{-1} p^{\prime}
$$

hence the projection $S_{W}$ of $\widehat{S}_{W, m}$ is just the free symplectic matrix

$$
S_{W}=\left[\begin{array}{cc}
L^{-1} Q & L^{-1} \\
P L^{-1} Q-L^{T} & P L^{-1}
\end{array}\right]
$$

generated by the quadratic form $W$. Note that if conversely $S$ is a free symplectic matrix

$$
S=\left[\begin{array}{ll}
A & B \\
C & D
\end{array}\right] \in S p(n), \quad \operatorname{det} B \neq 0
$$

then $S=S_{W}$ with $P=D B^{-1}, L=B^{-1}, Q=B^{-1} A$. Observe that the free symplectic $2 n \times 2 n$ matrices form a dense subset of $S p(n)$; this property will be used in the proof of Proposition 10.

The inverse $\widehat{S}_{W, m}^{-1}=\left(\widehat{S}_{W, m}\right)^{*}$ of $\widehat{S}_{W, m}$ is the operator $S_{W^{*}, m^{*}}$ where $W^{*}\left(x, x^{\prime}\right)=$ $-W\left(x^{\prime}, x\right)$ and $m^{*}=n-m, \bmod 4$. 


\subsection{Heisenberg-Weyl operators}

The operators $\widehat{T}\left(z_{0}\right)$ satisfy the metaplectic covariance formula:

$$
\widehat{S} \widehat{T}(z)=\widehat{T}(S z) \widehat{S} \quad(S=\pi(\widehat{S}))
$$

for every $\widehat{S} \in M p(n)$ and $z$. In fact, the metaplectic operators are the only unitary operators (up to a factor in $S^{1}$ ) satisfying (10):

For every $S \in S p(n)$ there exists a unitary transformation $\widehat{U}$ in $L^{2}\left(\mathbb{R}^{n}\right)$ satisfying (10) and $\widehat{U}$ is uniquely determined apart from a constant factor of modulus one.

The Heisenberg-Weyl operators moreover satisfy the relations

$$
\begin{gathered}
\widehat{T}\left(z_{0}\right) \widehat{T}\left(z_{1}\right)=e^{i \sigma\left(z_{0}, z_{1}\right)} \widehat{T}\left(z_{1}\right) \widehat{T}\left(z_{0}\right) \\
\widehat{T}\left(z_{0}+z_{1}\right)=e^{-\frac{i}{2} \sigma\left(z_{0}, z_{1}\right)} \widehat{T}\left(z_{0}\right) \widehat{T}\left(z_{1}\right)
\end{gathered}
$$

as is easily seen from their definition.

\subsection{Weyl operators}

Let $a^{w}=a^{w}(x, D)$ be the Weyl operator with symbol $a$ (which we always assume to belong to some suitable class, allowing the integrals to be viewed as distributions):

$$
a^{w} f(x)=\left(\frac{1}{2 \pi}\right)^{n} \iint e^{i\langle p, x-y\rangle} a\left(\frac{1}{2}(x+y), p\right) f(y) d^{n} y d^{n} p ;
$$

where $f \in \mathcal{S}\left(\mathbb{R}^{n}\right)$; equivalently

$$
a^{w}=\left(\frac{1}{2 \pi}\right)^{n} \int a_{\sigma}\left(z_{0}\right) \widehat{T}\left(z_{0}\right) d^{2 n} z_{0}
$$

where the "twisted symbol" $a_{\sigma}$ is the symplectic Fourier transform $F_{\sigma} a$ of $a$ :

$$
a_{\sigma}(z)=F_{\sigma} a(z)=\left(\frac{1}{2 \pi}\right)^{n} \int e^{-i \sigma\left(z, z^{\prime}\right)} a\left(z^{\prime}\right) d^{2 n} z^{\prime} .
$$

The compose $c^{w}=a^{w} \circ b^{w}$ (when defined) is the Weyl operator with twisted Weyl symbol

$$
c_{\sigma}=\left(\frac{1}{2 \pi}\right)^{n}\left(a_{\sigma} *_{\sigma} b_{\sigma}\right)
$$

where

$$
a *_{\sigma} b(z)=\int e^{\frac{i}{2} \sigma(z, u)} a(z-u) b(u) d^{2 n} u
$$

(see for instance Littlejohn [11, Wong [17]). 


\subsection{Generalized Fresnel Formula}

We will use the following formula, generalizing the usual Fresnel integral to complex Gaussians. Let $M$ be a real symmetric $m \times m$ matrix. If $M$ is invertible then the Fourier transform of the exponential $\exp (i\langle M x, x\rangle / 2)$ is given by the formula

$$
\left(\frac{1}{2 \pi}\right)^{m / 2} \int e^{-i\langle v, u\rangle} e^{\frac{i}{2}\langle M u, u\rangle} d^{m} u=|\operatorname{det} M|^{-1 / 2} e^{\frac{i \pi}{4} \operatorname{sgn} M} e^{-\frac{i}{2}\left\langle M^{-1} v, v\right\rangle}
$$

where $\operatorname{sgn} M$, the "signature" of $M$, is the number of $>0$ eigenvalues of $M$ minus the number of $<0$ eigenvalues.

For a proof of this formula see for instance [3, Appendix A.

\section{Discussion of the Mehlig-Wilkinson Formula}

In this Section we show that the Mehlig-Willkinson operators (31) indeed are metaplectic operators. We begin by giving two alternative expressions for these operators.

\subsection{Equivalent formulations}

We begin by remarking that the matrix

$$
M_{S}=\frac{1}{2} J(S+I)(S-I)^{-1}
$$

is symmetric; this immediately follows from the conditions

$$
S \in S p(n) \Longleftrightarrow S^{T} J S=J \Longleftrightarrow S J S^{T}=J .
$$

Notice that for every $M$ with $\operatorname{det}\left(M-\frac{1}{2} J\right) \neq 0$ the equation

$$
M=\frac{1}{2} J(S+I)(S-I)^{-1}
$$

can be solved in $S$, yielding

$$
S=\left(M-\frac{1}{2} J\right)^{-1}\left(M+\frac{1}{2} J\right) ;
$$

the relation $S \in S p(n)$ is then equivalent to $M$ being real and symmetric.

Lemma 1 Let $S \in S p(n)$ be such that $\operatorname{det}(S-I) \neq 0$. The operator

$$
\widehat{R}_{\nu}(S)=\left(\frac{1}{2 \pi}\right)^{n} \frac{i^{\nu}}{\sqrt{|\operatorname{det}(S-I)|}} \int e^{\frac{i}{2}\left\langle M_{S} z, z\right\rangle} \widehat{T}(z) d^{2 n} z
$$


can be written as

$$
\widehat{R}_{\nu}(S)=\left(\frac{1}{2 \pi}\right)^{n} i^{\nu} \sqrt{|\operatorname{det}(S-I)|} \int e^{-\frac{i}{2} \sigma(S z, z)} \widehat{T}((S-I) z) d^{2 n} z
$$

that is, as

$$
\widehat{R}_{\nu}(S)=\left(\frac{1}{2 \pi}\right)^{n} i^{\nu} \sqrt{|\operatorname{det}(S-I)|} \int \widehat{T}(S z) \widehat{T}(-z) d^{2 n} z
$$

Proof. We have

$$
\frac{1}{2} J(S+I)(S-I)^{-1}=\frac{1}{2} J+J(S-I)^{-1}
$$

hence, in view of the antisymmetry of $J$,

$$
\left\langle M_{S} z, z\right\rangle=\left\langle J(S-I)^{-1} z, z\right\rangle=\sigma\left((S-I)^{-1} z, z\right)
$$

Performing the change of variables $z \longmapsto(S-I) z$ we can rewrite the integral in the right-hand side of (16) as

$$
\begin{aligned}
\int e^{\frac{i}{2}\left\langle M_{S} z, z\right\rangle} \widehat{T}(z) d^{2 n} z & =\sqrt{|\operatorname{det}(S-I)|} \int e^{\frac{i}{2} \sigma(z,(S-I) z)} \widehat{T}((S-I) z) d^{2 n} z \\
& =\sqrt{|\operatorname{det}(S-I)|} \int e^{-\frac{i}{2} \sigma(S z, z)} \widehat{T}((S-I) z) d^{2 n} z
\end{aligned}
$$

hence (17). Taking into account the relation (12) we have

$$
\widehat{T}((S-I) z)=e^{\frac{i}{2} \sigma(S z, z)} \widehat{T}(S z) \widehat{T}(-z)
$$

and formula (18) follows.

Remark 2 Formulae (16) and (17)-(18) suggest that $\nu \pi$ could be a choice of $\pm \arg \operatorname{det}(S-I)$. This is however not the case (see (23) in Proposition 6 below); formula (26) will identify the integer $\nu$ with the Conley-Zehnder index.

Corollary 3 We have $\widehat{R}_{\nu}(S)=c_{S} \widehat{S}_{W, m}$ with $\left|c_{S}\right|=1$.

Proof. The operator $\widehat{R}_{\nu}(S)$ satisfies the metaplectic covariance relation

$$
\widehat{R}_{\nu}(S) \widehat{T}(z)=\widehat{T}(S z) \widehat{R}_{\nu}(S)
$$

as immediately follows from the alternative form (17) of $\widehat{R}_{\nu}(S)$. On the other hand, a straightforward calculation using formula (18) shows that $\widehat{R}_{\nu}(S)$ is unitary, hence the claim.

Let us precise Corollary 3 by discussing the choice of the constant $c_{S}$. 


\subsection{The case $\widehat{S}=\widehat{S}_{W, m}$}

We are going to show that the Mehlig-Wilkinson operators coincide with the metaplectic operators $\widehat{S}_{W, m}$ when $S=S_{W}$ and we will thereafter determine the correct choice for $\nu$; we will see that it is related by a simple formula to the usual Maslov index as defined in [4.

Let us first prove the following technical result:

Lemma 4 Let $S_{W}$ be a free symplectic matrix (9). We have

$$
\operatorname{det}\left(S_{W}-I\right)=(-1)^{n} \operatorname{det}(B) \operatorname{det}\left(B^{-1} A+D B^{-1}-B^{-1}-\left(B^{T}\right)^{-1}\right)
$$

that is, when $S$ is written in the form (8):

$$
\operatorname{det}\left(S_{W}-I\right)=(-1)^{n} \operatorname{det}\left(L^{-1}\right) \operatorname{det}\left(P+Q-L-L^{T}\right) .
$$

Proof. Since $B$ is invertible we can write $S-I$ as

$$
\left[\begin{array}{cc}
A-I & B \\
C & D-I
\end{array}\right]=\left[\begin{array}{cc}
0 & B \\
I & D-I
\end{array}\right]\left[\begin{array}{cc}
C-(D-I) B^{-1}(A-I) & 0 \\
B^{-1}(A-I) & I
\end{array}\right]
$$

and hence

$$
\operatorname{det}\left(S_{W}-I\right)=\operatorname{det}(-B) \operatorname{det}\left(C-(D-I) B^{-1}(A-I)\right) .
$$

Since $S$ is symplectic we have $C-D B^{-1} A=-\left(B^{T}\right)^{-1}$ (use for instance the fact that $\left.S^{T} J S=S J S^{T}=J\right)$ and hence

$$
\left.C-(D-I) B^{-1}(A-I)\right)=B^{-1} A+D B^{-1}-B^{-1}-\left(B^{T}\right)^{-1} ;
$$

the Lemma follows since $\operatorname{det}(-B)=(-1)^{n} \operatorname{det} B$.

Remark 5 The factorization (21) shows in particular that $\operatorname{ker}(S-I)$ is isomorphic to $\operatorname{ker}\left(P+Q-L-L^{T}\right)$ (cf. [13], Lemma 2.8, and proof of Lemma 2.9).

Let us denote by $W_{x x}$ the Hessian matrix of the function $x \longmapsto W(x, x)$, that is

$$
W_{x x}=P+Q-L-L^{T} .
$$

We have:

Proposition 6 Let $S=S_{W}$ be a free symplectic matrix (9) and $\widehat{R}_{\nu}(S)$ the corresponding Mehlig-Wilkinson operator. We have $\widehat{R}_{\nu}(S)=\widehat{S}_{W, m}$ provided that $\nu$ is chosen so that

$$
\nu \equiv m-\operatorname{Inert} W_{x x} \bmod 4
$$

in which case we have

$$
\frac{1}{\pi} \arg \operatorname{det}(S-I) \equiv-\nu+n \bmod 2
$$


Proof. Recall that we have shown that $\widehat{R}_{\nu}(S)=c_{S} \widehat{S}_{W, m}$ where $c_{S}$ is some complex constant with $\left|c_{S}\right|=1$. Let us determine that constant. Let $\delta \in$ $\mathcal{S}^{\prime}\left(\mathbb{R}^{n}\right)$ be the Dirac distribution centered at $x=0$; setting

$$
C_{W, \nu}=\left(\frac{1}{2 \pi}\right)^{n} \frac{i^{\nu}}{\sqrt{|\operatorname{det}(S-I)|}}
$$

we have, by definition of $\widehat{R}_{\nu}(S)$, writing $z_{0}=\left(x_{0}, p_{0}\right)$ in place of $z=(x, p)$ :

$$
\begin{aligned}
\widehat{R}_{\nu}(S) \delta(x) & =C_{W, \nu} \int e^{\frac{i}{2}\left\langle M_{S} z_{0}, z_{0}\right\rangle} e^{i\left(\left\langle p_{0}, x\right\rangle-\frac{1}{2}\left\langle p_{0}, x_{0}\right\rangle\right)} \delta\left(x-x_{0}\right) d^{2 n} z_{0} \\
& =C_{W, \nu} \int e^{\frac{i}{2}\left\langle M_{S}\left(x, p_{0}\right),\left(x, p_{0}\right)\right\rangle} e^{\frac{i}{2}\left\langle p_{0}, x\right\rangle} \delta\left(x-x_{0}\right) d^{2 n} z_{0}
\end{aligned}
$$

hence, setting $x=0$,

$$
\widehat{R}_{\nu}(S) \delta(0)=C_{W, \nu} \int e^{\frac{i}{2}\left\langle M_{S}\left(0, p_{0}\right),\left(0, p_{0}\right)\right\rangle} \delta\left(-x_{0}\right) d^{2 n} z_{0}
$$

that is, since $\int \delta\left(-x_{0}\right) d^{n} x_{0}=1$,

$$
\widehat{R}_{\nu}(S) \delta(0)=\left(\frac{1}{2 \pi}\right)^{n} \frac{i^{\nu}}{\sqrt{|\operatorname{det}(S-I)|}} \int e^{\frac{i}{2}\left\langle M_{S}\left(0, p_{0}\right),\left(0, p_{0}\right)\right\rangle} d^{n} p_{0} .
$$

Let us calculate the scalar product

$$
\left.\left\langle M_{S}\left(0, p_{0}\right),\left(0, p_{0}\right)\right\rangle=\sigma\left((S-I)^{-1} 0, p_{0}\right),\left(0, p_{0}\right)\right) .
$$

The relation $(x, p)=(S-I)^{-1}\left(0, p_{0}\right)$ is equivalent to $S(x, p)=\left(x, p+p_{0}\right)$ that is to

$$
p+p_{0}=\partial_{x} W(x, x) \text { and } p=-\partial_{x^{\prime}} W(x, x) .
$$

Using the explicit form (6) of $W$ together with Lemma 4 these relations yield

$$
x=\left(P+Q-L-L^{T}\right)^{-1} p_{0} \text { and } p=(L-Q)\left(P+Q-L-L^{T}\right)^{-1} p_{0}
$$

and hence

$$
\left\langle M_{S}\left(0, p_{0}\right),\left(0, p_{0}\right)\right\rangle=-\left\langle W_{x x}^{-1} p_{0}, p_{0}\right\rangle .
$$

Applying Fresnel's formula (15) we get

$$
\left(\frac{1}{2 \pi}\right)^{n} \int e^{\frac{i}{2}\left\langle M_{S}\left(0, p_{0}\right),\left(0, p_{0}\right)\right\rangle} d^{n} p_{0}=\left(\frac{1}{2 \pi}\right)^{n / 2} e^{-\frac{i \pi}{4} \operatorname{sgn} W_{x x}}\left|\operatorname{det} W_{x x}\right|^{1 / 2} ;
$$

noting that

$$
\frac{1}{\sqrt{\left|\operatorname{det}\left(S_{W}-I\right)\right|}}=|\operatorname{det} L|^{1 / 2}\left|\operatorname{det} W_{x x}\right|^{-1 / 2}
$$


(formula (20) in Lemma 4) we thus have

$$
\widehat{R}_{\nu}\left(S_{W}\right) \delta(0)=\left(\frac{1}{2 \pi}\right)^{n / 2} i^{\nu} e^{-\frac{i \pi}{4} \operatorname{sgn} W_{x x}}|\operatorname{det} L|^{1 / 2} .
$$

Now, by definition (5) of $\widehat{S}_{W, m}$,

$$
\widehat{S}_{W, m} \delta(0)=\left(\frac{1}{2 \pi}\right)^{n / 2} i^{m-n / 2}|\operatorname{det} L|^{1 / 2}
$$

hence $i^{\nu} e^{-\frac{i \pi}{4} \operatorname{sgn} W_{x x}}=i^{m-n / 2}$. It follows that we have

$$
\nu-\frac{1}{2} \operatorname{sgn} W_{x x} \equiv m-\frac{1}{2} n \bmod 4
$$

which is the same thing as (22) since $W_{x x}$ has rank $n$. In view of (19) we have

$$
\frac{1}{\pi} \arg \operatorname{det}\left(S_{W}-I\right)=n+m+\arg \operatorname{det} W_{x x} \bmod 2 ;
$$

formula (23) follows using (22).

Let us digress for a while on the integers $m$ and Inert $W_{x x}$ appearing in formula (22) and discuss them from the point of view of calculus of variations. It is for this purpose useful to recall that in Gutzwiller's formula (of which Mehlig and Wilkinson precisely want to give a new approach using the operators $\left.\widehat{R}_{\nu}(S)\right)$ the symplectic matrix $S$ is obtained from the monodromy matrix of an isolated Hamiltonian periodic orbit. Let us go a little bit further. Consider a Hamiltonian flow $\phi_{t}$ determined by some time-dependent Hamiltonian $H=H(z, t)$ defined on $\mathbb{R}_{z}^{2 n} \times \mathbb{R}_{t}$, and let $z_{0}=\left(x_{0}, p_{0}\right)$ be such that $\phi_{T}\left(z_{0}\right)=z_{0}$ for some $T>0$. The Jacobian matrices $S\left(z_{0}, t\right)=D \phi_{t}\left(z_{0}\right)$ are symplectic and satisfy the "variational equation"

$$
\frac{d}{d t} S\left(z_{0}, t\right)=J H^{\prime \prime}\left(z_{0}, t\right) S\left(z_{0}, t\right)
$$

where $H$ " $\left(z_{0}, t\right)$ is the Hessian matrix $D^{2} H\left(\phi_{t}\left(z_{0}\right), t\right)$. When $t$ varies from 0 to $T$ the matrices $S\left(z_{0}, t\right)$ describe a path in $S p(n)$ originating at the identity and ending at $S\left(z_{0}, T\right)$ (the "monodromy matrix"). Suppose that $S\left(z_{0}, T\right)$ is a free symplectic matrix $S_{W}$; then there exist $p$ and $p^{\prime}$ such that $\left(x_{0}, p\right)=S_{W}\left(x_{0}, p^{\prime}\right)$, that is, expressing $W$ in terms of $P, L, Q$ as in (8) and using (17), $p=\left(P-L^{T}\right) x_{0}$ and $p^{\prime}=(L-Q) x_{0}$, that is

$$
p-p^{\prime}=\left(P+Q-L-L^{T}\right) x_{0} .
$$

It thus appears (see for instance [13, 15]) that Inert $W_{x x}$ is Morse's [12] order of concavity of the periodic orbit through $z_{0}$. 
Remark 7 In [13] Piccione and his collaborators use the order of concavity, which they identify with Inert $W_{x x}$, to investigate the Maslov and Morse indices for periodic geodesics. The topic is also discussed at some length in MuratoreGinnaneschi [15] in connection with the study of Gutzwiller's formulae using field-theoretical methods.

Perhaps even more interesting is the relation between the index $\nu$ and an index defined by Conley and Zehnder in [2]. Let us denote by $S p_{0}(n)$ the set of all $S \in S p(n)$ such that $\operatorname{det}(S-I) \neq 0$. We have

$$
S p_{0}(n)=S p_{+}(n) \cup S p_{-}(n)
$$

where $S \in S p_{ \pm}(n)$ if and only if $\pm \operatorname{det}(S-I)>0$. The sets $S p_{ \pm}(n)$ are connected and every loop in $S p_{0}(n)$ is contractible in $S p(n)$. Consider now a path $\tilde{S}:[0, T] \longmapsto S p_{0}(n)$ going from the identity to $S=S(T)(S(T)$ may be viewed, if one wants, as the monodromy matrix of a periodic Hamiltonian orbit corresponding to a time-dependent Hamiltonian). The index of Conley and Zehnder associates to the path $\tilde{S}$ an integer $\mu_{C Z}(\tilde{S})$ only depending on the homotopy class (with fixed endpoints) of that path, and such that

$$
\operatorname{sign} \operatorname{det}(S-I)=(-1)^{n-\mu_{C Z}(\tilde{S})}
$$

that is, equivalently,

$$
\frac{1}{\pi} \arg \operatorname{det}(S-I) \equiv n-\mu_{C Z}(\tilde{S}) \bmod 2 .
$$

It follows from formula (23) in Proposition 6 that we have

$$
\nu \equiv \mu_{C Z}(\tilde{S}) \bmod 2
$$

and from formula (22) in the same proposition that

$$
\mu_{C Z}(\tilde{S}) \equiv m+n-\operatorname{Inert} W_{x x} \bmod 2 .
$$

\subsection{The general case}

Recall that we established in Lemma 4 the equality

$$
\operatorname{det}\left(S_{W}-I\right)=(-1)^{n} \operatorname{det} L^{-1} \operatorname{det}\left(P+Q-L-L^{T}\right) .
$$

valid for all free matrices $S_{W} \in S p(n)$. Also recall that every $\widehat{S} \in M p(n)$ can be written (in infinitely many ways) as a product $\widehat{S}=\widehat{S}_{W, m} \widehat{S}_{W^{\prime}, m^{\prime}}$. We are going to show that $\widehat{S}_{W, m}$ and $\widehat{S}_{W^{\prime}, m^{\prime}}$ always can be chosen such that $\operatorname{det}\left(S_{W}-I\right) \neq 0$ and $\operatorname{det}\left(S_{W^{\prime}}-I\right) \neq 0$. For that purpose we need the following straightforward factorization result, which we nevertheless glorify by putting it into italics: 
Lemma 8 Let $W$ be given by (6); then

$$
\widehat{S}_{W, m}=\widehat{V}_{-P} \widehat{M}_{L, m} \widehat{J} \widehat{V}_{-Q}
$$

where

$$
\widehat{V}_{-P} f(x)=e^{\frac{i}{2}\langle P x, x\rangle} f(x), \widehat{M}_{L, m} f(x)=i^{m} \sqrt{|\operatorname{det} L|} f(L x),
$$

and $\widehat{J}$ is the modified Fourier transform given by

$$
\widehat{J} f(x)=\left(\frac{1}{2 \pi i}\right)^{n / 2} \int e^{-i\left\langle x, x^{\prime}\right\rangle} f\left(x^{\prime}\right) d^{n} x^{\prime} .
$$

Proof. It is obvious using the explicit expression (6) of the quadratic form $W($ see 4]).

Let us now state and prove the first result of this section:

Proposition 9 (i) Every $\widehat{S} \in M p(n)$ can be written as a product

$$
\widehat{S}=\widehat{R}_{\nu}\left(S_{W}\right) \widehat{R}_{\nu^{\prime}}\left(S_{W^{\prime}}\right) .
$$

(ii) The Mehlig-Wilkinson operators thus generate $M p(n)$.

Proof. (ii) follows from (i) since the $\widehat{S}_{W, m}$ generate $M p(n)$. To prove (i) let us write $\widehat{S}=\widehat{S}_{W, m} \widehat{S}_{W^{\prime}, m^{\prime}}$ and apply (29) to each of the factors; letting $P^{\prime}, L^{\prime}, Q^{\prime}$ define $W^{\prime}$ just as (6) is defined by $P, L, Q$ we have

$$
\widehat{S}=\widehat{V}_{-P} \widehat{M}_{L, m} \widehat{J V} \widehat{-}_{-\left(P^{\prime}+Q\right)} \widehat{M}_{L^{\prime}, m^{\prime}} \widehat{J V} \widehat{V}_{-Q^{\prime}}
$$

We claim that $\widehat{S}_{W, m}$ and $\widehat{S}_{W^{\prime}, m^{\prime}}$ can be chosen in such a way that $\operatorname{det}\left(S_{W}-I\right) \neq$ 0 and $\operatorname{det}\left(S_{W^{\prime}}-I\right) \neq 0$ that is,

$$
\operatorname{det}\left(P+Q-L-L^{T}\right) \neq 0 \text { and } \operatorname{det}\left(P^{\prime}+Q^{\prime}-L^{\prime}-L^{\prime T}\right) \neq 0 ;
$$

this will prove the assertion in view of (28). We first remark that the right hand-side of (31) obviously does not change if we replace $P^{\prime}$ by $P^{\prime}+\lambda$ and $Q$ by $Q-\lambda$ where $\lambda \in \mathbb{R}$. Choose now $\lambda$ such that it is not an eigenvalue of $P+Q-L-L^{T}$ and $-\lambda$ is not an eigenvalue of $P^{\prime}+Q^{\prime}-L^{\prime}-L^{\prime T}$; then

$$
\operatorname{det}\left(P+Q-\lambda I-L-L^{T}\right) \neq 0 \text { and } \operatorname{det}\left(P^{\prime}+\lambda+Q^{\prime}-L-L^{T}\right) \neq 0
$$

and we have $\widehat{S}=\widehat{S}_{W_{1}, m_{1}} \widehat{S}_{W_{1}^{\prime}, m_{1}^{\prime}}$ with

$$
\begin{aligned}
& W_{1}\left(x, x^{\prime}\right)=\frac{1}{2}\langle P x, x\rangle-\left\langle L x, x^{\prime}\right\rangle+\frac{1}{2}\left\langle(Q-\lambda) x^{\prime}, x^{\prime}\right\rangle \\
& W_{1}^{\prime}\left(x, x^{\prime}\right)=\frac{1}{2}\left\langle\left(P^{\prime}+\lambda\right) x, x\right\rangle-\left\langle L^{\prime} x, x^{\prime}\right\rangle+\frac{1}{2}\left\langle Q^{\prime} x^{\prime}, x^{\prime}\right\rangle .
\end{aligned}
$$

So far, so good. But we haven't told the whole story yet: there remains to prove that $\widehat{S} \in M p(n)$ can be written in the form $\widehat{R}_{\nu}(S)$ if $\operatorname{det}(S-I) \neq 0$. 
Proposition 10 Let $\widehat{S} \in M p(n)$ be such that $\operatorname{det}(S-I) \neq 0$. If $\widehat{S}=$ $\widehat{R}_{\nu}\left(S_{W}\right) \widehat{R}_{\nu^{\prime}}\left(S_{W^{\prime}}\right)$ then $\widehat{S}=\widehat{R}_{\nu(S)}(S)$ with

$$
\nu(S)=\nu+\nu^{\prime}+n-\operatorname{Inert}\left(M+M^{\prime}\right)
$$

the matrices $M$ and $M^{\prime}$ being associated to $S$ and $S^{\prime}$ by formula (2).

Proof. A straightforward calculation using the composition formula (14) and the Fresnel integral (15) shows that

$$
\widehat{S}=\left(\frac{1}{2 \pi}\right)^{n} \frac{i^{\nu+\nu^{\prime}+\frac{1}{2} \operatorname{sgn}\left(M+M^{\prime}\right)}}{\sqrt{\left|\operatorname{det}\left(S_{W}-I\right)\left(S_{W^{\prime}}-I\right)\left(M+M^{\prime}\right)\right|}} \int e^{\frac{i}{2}\langle N z, z\rangle} \widehat{T}(z) d^{2 n} z
$$

where $M$ and $M^{\prime}$ correspond to $S_{W}$ and $S_{W^{\prime}}$ by (2) and

$$
N=M-\left(M+\frac{1}{2} J\right)\left(M+M^{\prime}\right)^{-1}\left(M-\frac{1}{2} J\right) .
$$

We claim that

$$
\operatorname{det}\left[\left(S_{W}-I\right)\left(S_{W^{\prime}}-I\right)\left(M+M^{\prime}\right)\right]=\operatorname{det}(S-I)
$$

(hence $M+M^{\prime}$ is indeed invertible), and

$$
N=\frac{1}{2} J(S+I)(S-I)^{-1}=M_{S} .
$$

Formula (34) is easy to check by a direct calculation: by definition of $M$ and $M^{\prime}$ we have, since $\operatorname{det} J=1$,

$$
\begin{aligned}
& \operatorname{det}\left[\left(S_{W}-I\right)\left(S_{W^{\prime}}-I\right)\left(M+M^{\prime}\right)\right]= \\
& \operatorname{det}\left[\left(S_{W}-I\right)\left(I+\left(S_{W}-I\right)^{-1}+\left(S_{W^{\prime}}-I\right)^{-1}\right)\left(S_{W^{\prime}}-I\right)\right]
\end{aligned}
$$

that is

$$
\operatorname{det}\left[\left(S_{W}-I\right)\left(S_{W^{\prime}}-I\right)\left(M+M^{\prime}\right)\right]=\operatorname{det}\left(S_{W} S_{W^{\prime}}-I\right)
$$

which is precisely (34). Formula (35) is at first sight more cumbersome, and one might be tempted to use the oscillator semigroup calculations of Howe [10] at this stage. There is however an easier way out: assume that $\widehat{S}=\widehat{S}_{W^{\prime \prime}, m^{\prime \prime}}$; we know by Proposition 9 that we must have in this case

$$
N=\frac{1}{2} J\left(S_{W} S_{W^{\prime}}+I\right)\left(S_{W} S_{W^{\prime}}-I\right)^{-1}
$$

and this algebraic identity then holds for all $S=S_{W} S_{W^{\prime}}$ since the free symplectic matrices are dense in $S p(n)$. Formula (33) can thus be rewritten

$$
\widehat{S}=\left(\frac{1}{2 \pi}\right)^{n} \frac{i^{\nu+\nu^{\prime}+\frac{1}{2} \operatorname{sgn}\left(M+M^{\prime}\right)}}{\sqrt{|\operatorname{det}(S-I)|}} \int e^{\frac{i}{2}\left\langle M_{S} z, z\right\rangle} \widehat{T}(z) d^{2 n} z
$$


and formula (32) follows noting that if $R$ is any real invertible $2 n \times 2 n$ symmetric matrix with $q$ negative eigenvalues we have $\arg \operatorname{det} R=q \pi \bmod 2 \pi$ and $\frac{1}{2} \operatorname{sgn} R=n-q$ and hence

$$
\frac{1}{2} \operatorname{sgn}\left(M+M^{\prime}\right)=n-\operatorname{Inert}\left(M+M^{\prime}\right) .
$$

\section{Concluding Remarks}

We have justified Mehlig and Wilkinson's claim that the metaplectic operators corresponding to symplectic matrices with no eigenvalues equal to one can be written in the form (3); we have in addition shown that every metaplectic operator can be written as the product of exactly two such operators. There are however still interesting open problems. It would be interesting to relate the index $\nu$ appearing in (3) to the cohomological Maslov index on $M p(n)$ we constructed in [4, 5]: this would certainly lead to simpler -or at least more tractable- calculations for the indices intervening in Gutzwiller's formula, as already demonstrated in our previous paper [7] where we examined the Maslov index of the monodromy matrix associated to a periodic Hamiltonian orbit. As shown by (26) a related mathematical problem would be to express the Conley-Zehnder index in terms of Leray's index studied in de Gosson [6].

We hope to come back to these important and interesting questions in a near future, together with applications to various trace formulae.

Acknowledgement 11 I wish to thank Keith Hannabuss for having drawn my attention to his work and having pointed out the relationship between it and the Mehlig-Wilkinson constructions. I also extend my warmest thanks to the referees for valuable suggestions and for having pointed out some miscalculations in an early version of the manuscript.

Acknowledgement 12 This work has been partially supported by a grant of the Max-Planck-Institut fuer Gravitationsphysik (Albert-Einstein-Institut, Golm). I wish to thank Prof. Hermann Nicolai for his kind hospitality.

\section{References}

[1] D. Bowes and K. Hannabuss. Weyl quantization and star products. J. Geom. Phys. 22, 319-348, 1997.

[2] C. Conley and E. Zehnder. Morse-type index theory for flows and periodic solutions of Hamiltonian equations. Comm. Pure and Appl. Math. 37:207-253, 1984. 
[3] G. B. Folland. Harmonic Analysis in Phase space. Annals of Mathematics studies, Princeton University Press, Princeton, N.J., 1989.

[4] M. de Gosson. Maslov Indices on $M p(n)$. Ann. Inst. Fourier, Grenoble, 40(3) (1990) 537-55.

[5] M. de Gosson. Cocycles de Demazure-Kashiwara et Géométrie Métaplectique. J. Geom. Phys. 9:255-280, 1992.

[6] M. de Gosson. The structure of $q$-symplectic geometry. J. Math. Pures et Appl. 71, 429-453, 1992.

[7] M. de Gosson and S. de Gosson. The Maslov Index indices of Periodic Hamiltonian Orbits (with S. de Gosson). J. Phys. A: Math. Gen. 36(48) 615-622. 2003

[8] K. C. Hannabuss. Characters and contact transformations. Math. Proc. Camb. Phil. Soc. 90, 465-476, 1981.

[9] M. C. Gutzwiller. Chaos in Classical and Quantum Mechanics. Interdisciplinary Applied Mathematics, Springer-Verlag, 1990.

[10] R. Howe. The Oscillator Semigroup. Proc. of Symposia in Pure Mathematics 48, Amer. Math. Soc. 61-132, 1988.

[11] R. G. Littlejohn. The semiclassical evolution of wave packets. Physics Reports 138(4-5):193-291, 1986.

[12] M. Morse. The Calculus of Variations in the Large. AMS, Providence, R. I., 1935.

[13] R. C. Nostre-Marques, P. Piccione, and D. V. Tausk. On the Morse and the Maslov index for periodic geodesics of arbitrary causal character. Differential Geometry and its Applications, Proc. Conf. Opava, August 27-31, 2001, 343-358.

[14] B. Mehlig and M. Wilkinson. Semiclassical trace formulae using coherent states. Ann. Phys. 18(10), 6-7, 541-555, 2001.

[15] P. Muratore-Ginnaneschi. Path integration over closed loops and Gutzwiller's trace formula. Phys. Rep. 383 299-397, 2003.

[16] M. Wilkinson. Wannier functions for lattices in a magnetic field. J. Phys.: Condens. Matter 10:7407-7427, 1998.

[17] M. W. Wong. Weyl Transforms. Springer, 1998. 